\title{
Vertical phase separation in small molecule:polymer blend organic thin film transistors can be dynamically controlled
}

Kui Zhao ${ }^{1}$, Olga Wodo, ${ }^{2,6}$, Dingding Ren ${ }^{1}$, Hadayat Ullah Khan ${ }^{1,7}$, Muhammad Rizwan Niazi ${ }^{1}$, Hanlin Hu ${ }^{l}$, Maged Abdelsamie ${ }^{l}$, Ruipeng $\mathrm{Li}^{1}$, Erqiang $\mathrm{Li}^{3}$, Liyang $\mathrm{Yu}^{l}$, Buyi Yan ${ }^{1}$, Marcia M. Payne $^{4}$, Jeremy Smith ${ }^{5}$, John E. Anthony ${ }^{4}$, Thomas D. Anthopoulos ${ }^{5}$, Sigurdur Thoroddsen ${ }^{3}$, Baskar Ganapathysubramanian ${ }^{2}$, Aram Amassian ${ }^{1 *}$

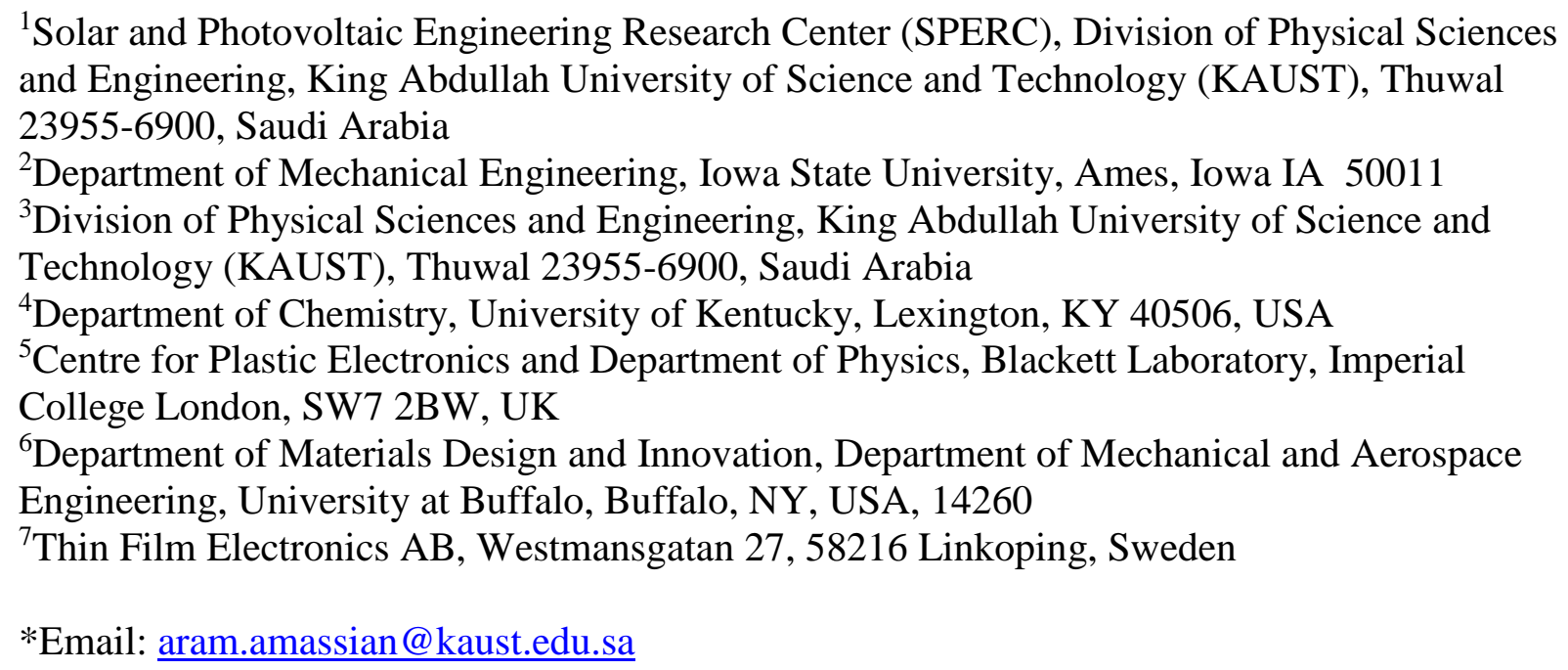

\section{Abstract:}

Blending of small-molecule organic semiconductors (OSCs) with amorphous polymers is known to yield high performance organic thin film transistors (OTFTs). Vertical stratification of the OSC and polymer binder into well-defined layers is crucial in such systems and their vertical order determines whether the coating is compatible with a top and/or a bottom gate OTFT configuration. Here, we investigate the formation of such blends prepared via spincoating in conditions which yield bilayer and trilayer stratifications, and use a combination of experimental and computational tools to study the competing effects of formulation thermodynamics and process kinetics in mediating the final vertical stratification. We show that trilayer stratification (OSC/polymer/OSC) is the thermodynamically favored configuration and that formation of the buried OSC layer can be kinetically inhibited in certain conditions of spincoating, resulting in a bilayer stack instead. Our analysis reveals that preferential loss of the 
OSC, combined with early aggregation of the polymer phase due to rapid drying, inhibit the formation of the buried OSC layer. We then moderate the fluid dynamics and drying kinetics during spin-coating to promote trilayer stratification with a high quality buried OSC layer which yields unusually high mobility $>2 \mathrm{~cm}^{2} \mathrm{~V}^{-1} \mathrm{~s}^{-1}$ in the bottom-gate top-contact configuration.

Keywords: organic thin film transistors; polymer-molecule blends; vertical phase separation; phase field simulations; in situ UV-Vis absorption

\section{Introduction}

Organic thin-film transistors (OTFTs) show ever-increasing possibility for low-cost, large-scale solution-based manufacturing of flexible electronic devices thanks to remarkable improvements of the carrier mobility, now well beyond $5 \mathrm{~cm}^{2} / \mathrm{Vs}^{[1-7]}$ Small molecule organic semiconductors (OSCs) which exhibit high carrier mobility are usually characterized by continuous thin films exhibiting a high degree of crystallinity, lamellar texture purity and an inplane packing motif which favors two-dimensional charge transport. ${ }^{[8-11]}$ Achieving continuous thin film formation via solution processing can be challenging due to molecular shape and packing anisotropies and a propensity to dewet. ${ }^{[12-13]}$ Forming films with excellent substrate coverage and lamellar texture also requires crystallization to be initiated from the solid-liquid interface ${ }^{[14]}$ while slight fluctuations in drying conditions and extended solvent vapor exposure can strongly impact the film formation and degree of crystallinity. ${ }^{[15,16]}$

Blending the small molecule OSC with a polymer has recently emerged as an effective way to improve the processability, reproducibility and stability of small molecule OTFTs, without sacrificing the intrinsic mobility of the OSC. ${ }^{[12,17-19]}$ This is largely due to the ability of the blend to phase separate into a layered structure which conserves the continuity, crystallinity, and molecular packing of the small molecule OSC, and even improving it in some instances. ${ }^{[13,19-21]}$ The current understanding of the correlation between solution-casting 
conditions and the resulting morphology in terms of phase separation and crystallization of the OSC within the blends, as well as the corresponding device performance is still in its infancy for small molecule-polymer blends, but some of the desirable features are clearly identified. For instance, phase separation should lead to the formation of continuous stratified layer(s) of highly crystalline small molecule OSC forming large, interconnected crystalline domains exhibiting lamellar texture with two-dimensional in-plane packing motif across the channel of the transistor, not unlike the neat OSC devices.

The formation of stratified structures is thought to be influenced by surface tension and wetting, as well as by crystallization and phase separation. ${ }^{[22-24]}$ Phase separation in blends proceeds either via the nucleation and growth of one phase in a surrounding medium or by the spontaneous (spinodal) route because of miscibility of the two components. ${ }^{[22]}$ In some instances, when phase separation happens quickly, preceeding wetting, such blends result in nanoscale phase separation as in the classical case of polymer:fullerene bulk heterojunction organic solar cells. ${ }^{[25]}$ Vertical phase separation, including stratification, occurs when the formation of wetting layers is given sufficient time to progress without the onset of bulk phase separation. Indeed, some correlation has been suggested between solution-casting conditions and morphology in the case of spin-cast blends. For example, the formation of a polycrystalline diF-TES-ADT layer on top of PMMA is related to the solvent evaporation rate, which determines whether to form a bi-layer structure or a de-wetted film. ${ }^{[26]}$ Ohe et al.reported TIPSpentacene accumulation and stratification at both interfaces with poly $(\alpha$-methyl styrene $)$ (PaMS) in the middle, pointing to the influence of the substrate on the phase separation outcome ${ }^{[18]}$ The nature of the polymer structure also plays a role in mediating the vertical phase separation. Blending TIPS-pentacene with a semi-crystalline isotactic polystyrene (i-PS) was reported to form stratification with small molecules segregating to the interfaces; ${ }^{[17]}$ whereas semi-crystalline syndiotactic polystyrene (s-PS) was found to inhibit the segregation and crystallization of diF-TESADT. ${ }^{[27]}$ Smith et al. further found that amorphous semi-conducting 
polymer could mediate conduction across grain boundaries in diF-TES-ADT-rich regions when the diF-TES-ADT layer crystallizes on top of the poly(triarylamine) (PTAA) or poly(dialkylfluorene-co-dimethyl-triarylamine) (PF-TAA), for bottom-contact top-gate (BCTG) devices. ${ }^{[1,7]}$ The BCTG device structure, which relies on an efficient pathway for charge transport on the semiconductor top surface, shows carrier mobility of greater than $5 \mathrm{~cm}^{2} \mathrm{~V}^{-1} \mathrm{~s}^{-1}$ from a device fabricated by a simple two-step spin-casting process. ${ }^{[1]}$

However, bi-layer vertical stratification is not suitable for all device configurations, such as bottom-contact bottom-gate (BCBG), or bottom-gate top-contact (BGTC). The ability to control the formation of a buried diF-TES-ADT layer beneath the polymer layer, such as PTAA, is of great interest; more generally, understanding which processing factors, if any, mediate the vertical stratification is of value. A great deal of effort has been spent to control the vertical phase separation, including by controlling the surface energy of the substrate, the choice of solvent and the polymer characteristics. However, one particularly interesting question is whether the solution processing method itself can be used to control the phase separation behavior, especially given the significant mismatch in physicochemical properties of the blend components, including their solubilities and viscosities. Clearly there could be tremendous scientific and technological benefit in understanding how processing conditions mediate stratification. This will enable identifying control strategies to optimize processes like spincoating, and the ability to extend this to more scalable processes.

Here, we investigate the spin-casting of blends of a small molecule OSC (diF-TESADT) and amorphous polymer (PTAA) under conditions yielding different vertical stratification profiles, with the aim of identifying how processing parameters can be leveraged to mediate or engineer the vertical stratification to achieve desired functionality. We utilize a combination of in situ optical reflectometry and in situ UV-visible absorption to investigate thinning/drying kinetics of the solution, phase transformation kinetics of the OSC and polymer components, and the solution state of the solute during spin-casting. We utilized our 
computational framework $^{[28-30]}$ that models morphology evolution during solution-based fabrication of organic thin films. The computational results are highly consistent with experimental observation and reveal the importance of liquid-liquid phase separation, preferential ejection of diF-TES-ADT, and drying kinetics. All these factors affect phase separation behavior during solution-casting and impact the final vertical stratification of the solid-state blend. We computationally identify the kinetic conditions which promote trilayer stratification and utilize this insight to process a highly ordered and contiguous buried OSC layer which yields carrier mobility $>2 \mathrm{~cm}^{2} \mathrm{~V}^{-1} \mathrm{~s}^{-1}$ in bottom-gate top-contact (BGTC) OTFTS.

\section{Results and discussion}

\subsection{Polymer-molecule blend OTFTs with bilayer and trilayer vertical stratification}

We fabricated diF-TES-ADT:PTAA blend OTFTs by spin-coating, using a recipe inspired from a previously reported process. ${ }^{[1]}$ The recipe consists of two steps, starting with a slower and shorter first step (700 rpm for $10 \mathrm{~s}$ ), followed by a second longer and faster step lasting $20 \mathrm{~s}$ and ranging in speed from $1000 \mathrm{rpm}$ to $5000 \mathrm{rpm}$. We have fabricated BGTC and BCTG OTFTs as a means of indirectly probing the formation, respectively, of a buried OSC layer beneath the polymer layer, or on top of the polymer layer. The transfer and output currentvoltage characteristics are shown in Figure $\mathbf{S 1}$ and 2. The saturation mobilities extracted for BGTC devices decrease slightly from $0.12 \mathrm{~cm}^{2} \mathrm{~V}^{-1} \mathrm{~s}^{-1}$ to $0.07 \mathrm{~cm}^{2} \mathrm{~V}^{-1} \mathrm{~s}^{-1}$ with increasing speed from $1000 \mathrm{rpm}$ to $3000 \mathrm{rpm}$ (Figure 1a). These mobilities are slightly higher than those of neat diF-TES-ADT prepared by spin coating from toluene $\left(0.037 \mathrm{~cm}^{2} \mathrm{~V}^{-1} \mathrm{~s}^{-1}\right)$ and similar to previous reports by Gundlach et al., ${ }^{[6]}$ indicating that a buried OSC layer forms beneath the PTAA. At higher speeds of $4000 \mathrm{rpm}$ and $5000 \mathrm{rpm}$, mobility falls drastically to $\sim 10^{-4} \mathrm{~cm}^{2} \mathrm{~V}^{-1} \mathrm{~s}^{-1}$, approaching the performance of neat PTAA $\left(\sim 10^{-5} \mathrm{~cm}^{2} \mathrm{~V}^{-1} \mathrm{~s}^{-1}\right.$, dark dashed line in Figure 1a), suggesting at the very least the loss of continuity of the buried OSC layer. By contrast, the mobilities of BCTG devices show a non-monotonic dependence on speed with mobilities 
varying in the range of $0.94-1.68 \mathrm{~cm}^{2} \mathrm{~V}^{-1} \mathrm{~s}^{-1}$, indicating the OSC overlayer is not compromised. The BGTC and BCTG devices provide potentially significant hints that spin-coating processing conditions influence blend layer stratification.

To shed light directly on stratification, we performed cross-sectional energy-filtered transmission electron microscopy (EFTEM) on blend films (see Figure S3 and $\mathbf{4}$ for more details of crystalline texture and surface morphology). Elemental maps focusing on the carbon $(284 \mathrm{eV})$ and sulfur $(165 \mathrm{eV})$ signals associated predominantly to PTAA and diF-TES-ADT rich phases locate the respective components (Figure 1b). The micrographs match our hyphothesis about vertical stratification of the buried diF-TES-ADT layer and reveal the presence of bilayer versus trilayer structures. A trilayer structure of diF-TES-ADT/PTAA/diFTES-ADT with abrupt interfaces is observed at low speed of $1000 \mathrm{rpm}$, where the high quality buried diF-TES-ADT layer accounts for the high mobility of BGTC device. At the intermediate speed (3000 rpm), the buried diF-TES-ADT layer becomes much thinner than the top diF-TESADT layer. The buried layer disappears entirely for films prepared at high speed (5000 rpm) resulting in a bilayer stratification with diF-TES-ADT layer atop PTAA. This observation of diminished buried diF-TES-ADT layer is consistent with the trend of significant drop of mobility for BGTC device. 


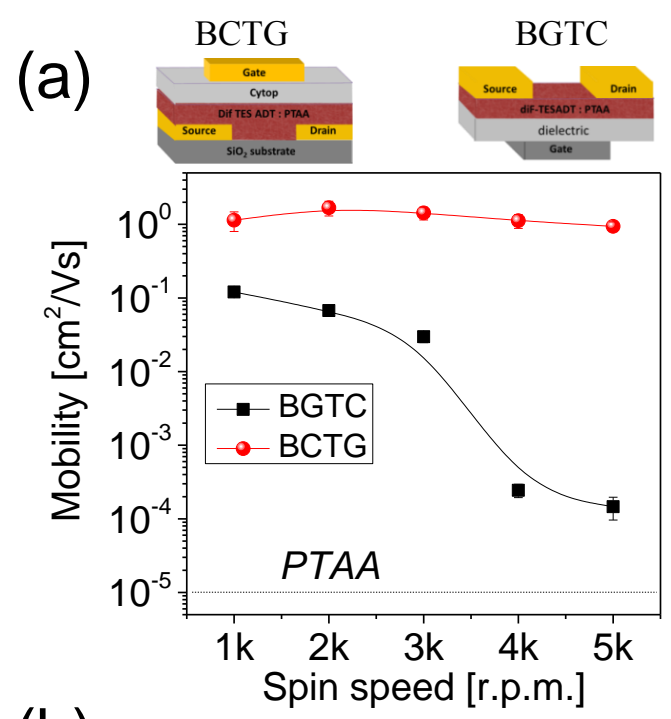

(c)

(b)

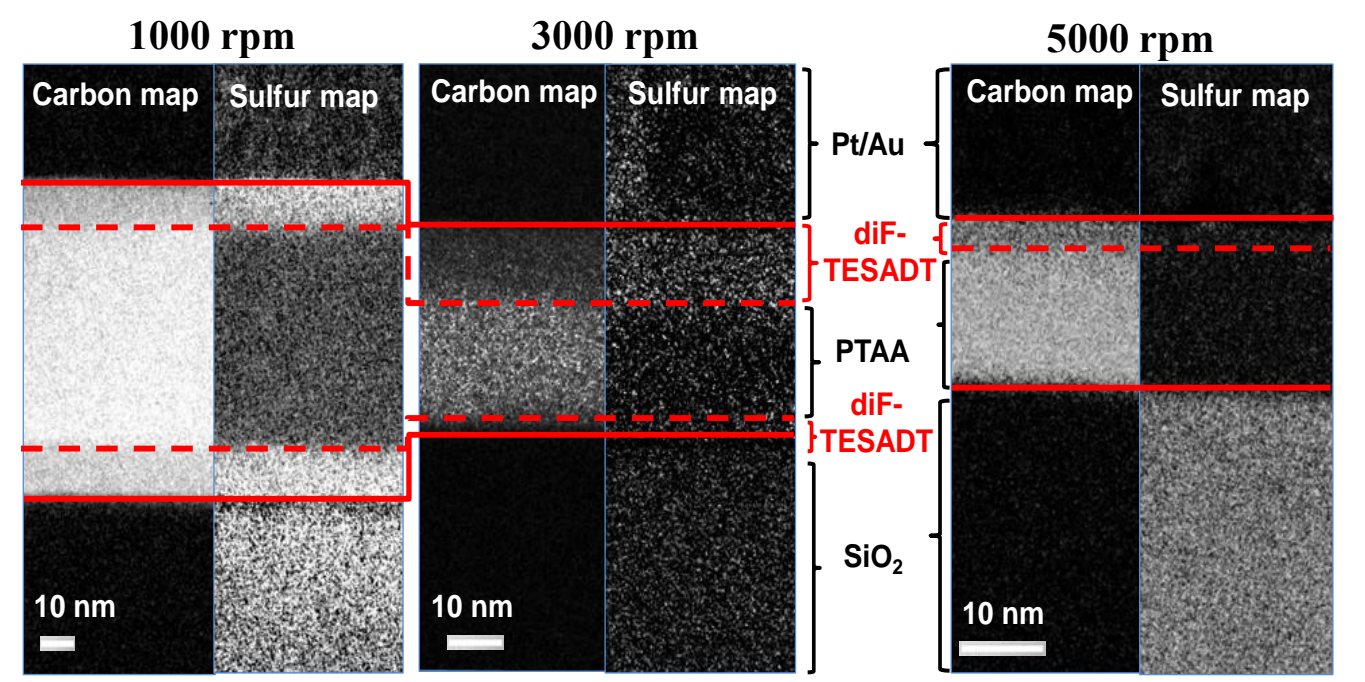

Figure 1. (a) Evolution of bottom-gate top-contact (BGTC) and bottom-contact top-gate (BCTG) field-effect mobilities of diF-TES-ADT:PTAA blend OTFTs with respect to spincoating speed. The mobility of neat PTAA film (dashed line, spin-cast from tetralin, $\sim 10^{-5}$ $\mathrm{cm}^{2} \mathrm{~V}^{-1} \mathrm{~s}^{-1}$ ) is shown for comparison. (b) Cross-sectional energy-filtered transmission electron micrographs (EFTEM) of diF-TES-ADT:PTAA blend films processed using spin-speeds of 1000, 3000, and $5000 \mathrm{rpm}$. The carbon mapping (284 eV) and sulfur mapping (165 eV) reveal the distribution of PTAA rich layer and diF-TES-ADT rich layer, respectively. (c) Summary of the thickness and vertical stratification of diF-TES-ADT and PTAA components. 
We observe a sharp drop in the thickness of the overall blend film as well as changing OSC to polymer thickness ratio with increasing spin-speed (Figure 1c). With increase in spinspeed (from $1000 \mathrm{rpm}$ to $3000 \mathrm{rpm}$ and $5000 \mathrm{rpm}$ ), we observe a drastic and continuous reduction in the thickness of the buried OSC from $\sim 15 \mathrm{~nm}$ to $\sim 4 \mathrm{~nm}$ and $0 \mathrm{~nm}$, consistent with drastic loss of carrier mobility in BGTC devices. Meanwhile the top OSC layer thickness varies slightly from $\sim 12 \mathrm{~nm}$ to $\sim 13 \mathrm{~nm}$ and $\sim 8 \mathrm{~nm}$, in line with the observation of consistently high mobility in BCTG devices. The PTAA thickness decreases from $\sim 60 \mathrm{~nm}$ to $\sim 34 \mathrm{~nm}$ and $\sim 32$ $\mathrm{nm}$, nearly by half from 1000 to $3000 \mathrm{rpm}$, with little to no change from 3000 to $5000 \mathrm{rpm}$. The volume ratio of the OSC and polymer components is estimated from these measurements. The initial component volume ratio in solution was nearly 45:55 (OSC:polymer). Increasing the spin-speed from $1000 \mathrm{rpm}$ to $5000 \mathrm{rpm}$ reduces the overall OSC thickness to $1 / 3$, whereas PTAA layer thickness decreases by $1 / 2$. These observations indicate the OSC and polymer components may be lost at different rates during the spin-coating process, most likely as a result of ejection and flow during the second spin-coating step.

\subsection{In-situ investigation of the spin-coating process}

We now seek to investigate the solution-thinning and molecular aggregation during spin-coating of the tetraline-based solution. To track the evolution of solution thickness, we performed in situ spectroscopic reflectometry and ellipsometry measurements during spincoating $^{[31]}$ (see Figure S5 for schematic of the in situ setup). The thinning data reveal that the solvent fully dries in $60 \mathrm{~s}$ at high spin speed $(5000 \mathrm{rpm})$ but requires $138 \mathrm{~s}$ at the lowest speed (1000 rpm) (see Figure S6 for more details). This drying time is substantially longer than what was previously reported in the contexts of small molecule OSCs using toluene $(\sim 8-10 \mathrm{~s}$; at room temperature) and polymer:fullerene bulk heterojunction layers using chlorobenzene ( $7-$ $8 \mathrm{~s}$; at an elevated temperature). ${ }^{[32,33]}$ Importantly, the spin-coating conditions explored in this study do not dry the solution at the 30 second mark, indicating that the film formation process 
is not quenched during spin-coating. Instead, the spinning process appears to be managing the time-evolution of the volume, composition and evaporation rate of the solution. ${ }^{[34]}$ We plot in Figure 2a the evolution of the solution thickness calculated from the combined optical measurements throughout the $30 \mathrm{~s}$ duration of spin-coating, including the increase of spin-speed from $700 \mathrm{rpm}$ at the $10 \mathrm{~s}$ mark. We observe a rapid decrease of the solution thickness from ca. $7 \mu \mathrm{m}$ at $10 \mathrm{~s}$ to ca. $3.1 \mu \mathrm{m}(0.6 \mu \mathrm{m})$ upon accelerating to $1000 \mathrm{rpm}(5000 \mathrm{rpm})$, which we attribute to ejection and/or outward flow. The resulting wet thickness decreases fivefold, whereas the total dry thickness reported in Figure 1c decreases only by a factor of ca. 2.175, indicating a complicated relationship between the solution processing conditions and concentration the solute composition of the wet film at $30 \mathrm{~s}$, when flow/ejection losses is halted. We estimate a relative solution volume loss of $25-80 \%$ after $10 \mathrm{~s}$ upon increasing the spin-speed to $1000-5000$ rpm, respectively (Figure $\mathbf{2 b}$, inset).

Time-resolved UV-Vis absorption measurements were performed during spin-coating with the aim of monitoring the vibronic progressions of the solute components for evidence of aggregation or solidification during the dynamic spin-coating conditions (Figure 2b). ${ }^{[32]}$ The absorption spectra of the blend solutions exhibit a sharp peak at ca. $526 \mathrm{~nm}$ and a broader peak at ca. $383 \mathrm{~nm}$, assigned to the characteristic singlet transition of solvated diF-TES-ADT ${ }^{[27]}$ and PTAA,${ }^{[35]}$ respectively. As expected, both absorption features decrease in intensity during the $30 \mathrm{~s}$ spin-casting experiments indicating loss of solute material from the light path, but do not exhibiting any shift in the peak position expected with solid state formation (Figure S7). The latter observation indicates that diF-TES-ADT remains mostly dissolved and un-aggregated throughout the spin-coating process, ${ }^{[27]}$ which is also confirmed by in situ high-speed polarized optical micrographs (HSPOM) taken during spin-coating ${ }^{[36]}$ (Figure S8).

The time-dependent decrease of absorption associated to the two solute components can be monitored to track whether the solute composition changes during the dynamic spin-casting process, as expected from ex situ observations made in Figure 1. We have traced the evolution 
of $\mathrm{A}_{526}$ and $\mathrm{A}_{383}$, absorption intensities of peaks at ca. $526 \mathrm{~nm}$ and ca. $383 \mathrm{~nm}$ for diF-TESADT and PTAA, respectively (Figure S9). We find the intensity of $\mathrm{A}_{383}$ decreases toward the dry-film absorption during the spin-coating process for spin-speeds of $3000 \mathrm{rpm}$ and more, indicating early aggregation of PTAA, as early as ca. $25 \mathrm{~s}$ for $3000 \mathrm{rpm}$ and ca. $20 \mathrm{~s}$ for 5000 rpm. These measurements suggest that PTAA aggregates at the solid-liquid interface, which prevents any further loss of PTAA, in agreement with EFTEM observations (Figure 1b). Another possible explanation is that the PTAA and diF-TES-ADT undergo liquid-liquid vertical phase separation, which drives the higher viscosity PTAA solution toward the substrate and resists the outward flow of the bulk solution. By contrast, the uninhibited decrease of $A_{526}$ for all speeds suggests diF-TES-ADT is continuously lost from the light path, resulting in dynamical changes of both concentration and solute composition.

To highlight the differential loss of the solute components, we have plotted the timeevolution of $\mathrm{A}_{526} / \mathrm{A}_{383}$ during the spin-coating process (Figure 2c). The results clearly show evidence of a continuous decrease in $\mathrm{A}_{526} / \mathrm{A}_{383}$ during the entire spin-casting process, which provides evidence of preferential loss of diF-TES-ADT. It is noteworthy that the preferential ejection of diF-TES-ADT is magnified significantly for higher spin-speeds, as evidenced by rapid rate of decrease of $\mathrm{A}_{526} / \mathrm{A}_{383}$. In actuality, we estimate that the preferential loss of diFTES-ADT results in an increase in the PTAA volume fraction in the wet film at $30 \mathrm{~s}$, increasing from ca. $1.9 \%$ to ca. $2.5 \%$ and ca. $3.0 \%$ when the second stage spin-speed increases from 1000 rpm to $3000 \mathrm{rpm}$ and $5000 \mathrm{rpm}$ (Figure 1c). This higher concentration may be the cause for earlier aggregation of PTAA. 
(a)

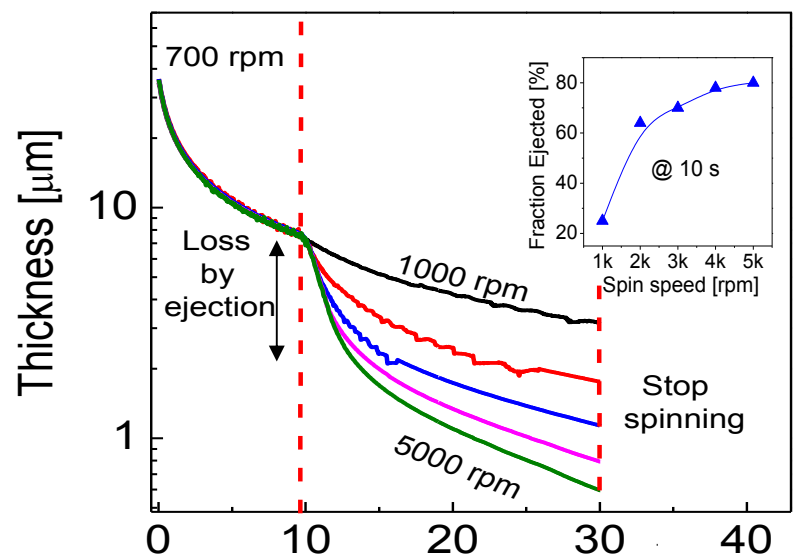

(b)

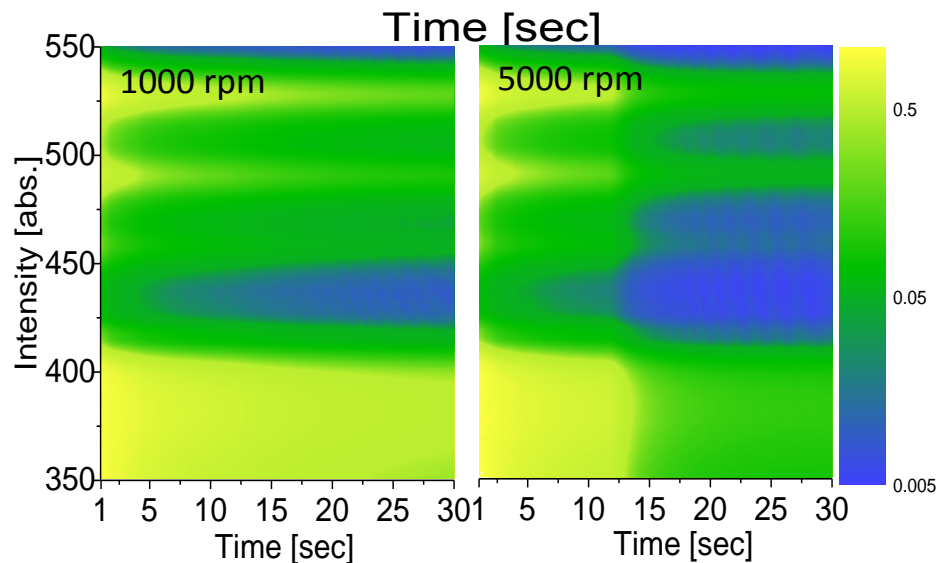

(c)

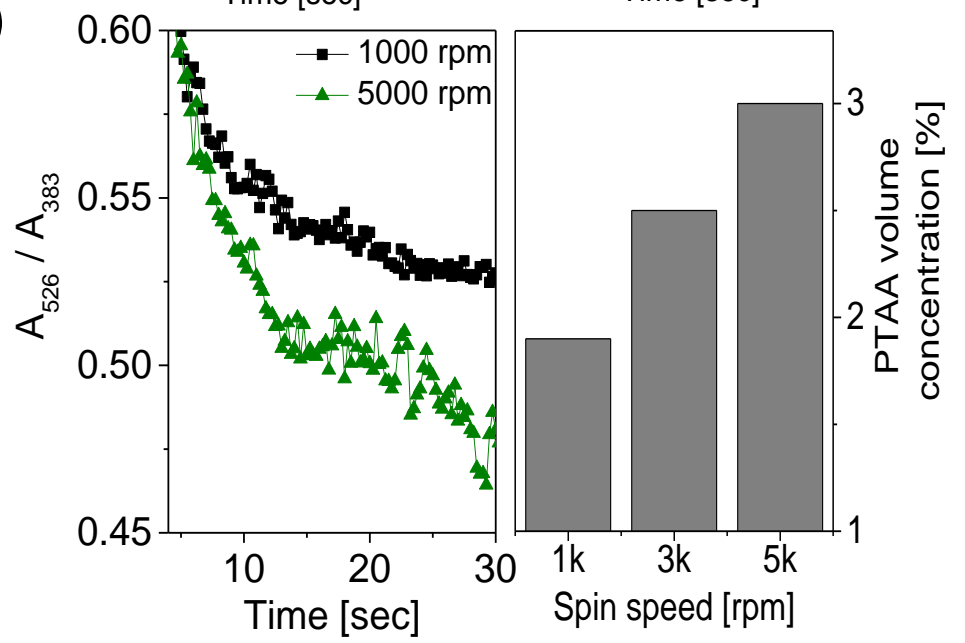

Figure 2. (a) Solution thinning behavior obtained from time-resolved spectroscopic reflectometry (TRSR). Inset showing the volume fraction of the ejected solution at different spin-speeds. (b) The UV-vis absorption spectra of diF-TES-ADT:PTAA blend solutions during spin-casting processed at $1000 \mathrm{rpm}$ and $5000 \mathrm{rpm}$. The scale bar on the right shows the absorption intensity. (c) Time-resolved peak intensity ratio of diF-TES-ADT and PTAA phases 
$\left(\mathrm{A}_{526} / \mathrm{A}_{383}\right)$ during spin-casting processed at spin-speeds of $1000 \mathrm{rpm}$ and $5000 \mathrm{rpm}$ (left), associated with the relevant PTAA volume fraction in the as-cast wet films (right).

\subsection{Modeling evaporation induced morphology evolution during spin-coating}

The experimental results presented thus far provide us with direct evidence that diFTES-ADT is preferentially lost during spin-coating, a fact significantly accentuated by the dynamic change of the spin-speed at $10 \mathrm{~s}$. We now turn our attention to understanding how such phenomena dynamically modulate the vertical stratification such that it may be varied from a trilayer at low speed to a bilayer at higher speed. To do so, we deploy a computational framework for morphology evolution, previously reported by Wodo et al., ${ }^{[30]}$ to study evaporation and centrifugal force mediated phase separation. The framework takes into account the physicochemical properties of the ternary solution, and the evaporation rate, $\mathrm{k}_{\mathrm{e}}$, of the solvent from the top surface, as these factors control the phase separation in blend systems. ${ }^{[37]}$ The rate at which solvent is removed from the top layer depends on various factors (e.g. solvent volatility, spinning velocity during spin-coating). During evaporation, the initially dilute solution becomes enriched in the two solutes due to the depletion of solvent. The loss of solvent results in an increased interaction between the solutes and triggers morphology evolution. The evolution is critically determined by the evaporation rate and diffusion of solutes (and solvent) within the film. As the solvent is removed, the blend is pushed into the spinodal range (immiscible conditions) and undergoes phase separation. Under these conditions, the solution is unstable and even small concentration fluctuations are rapidly amplified resulting in phase separation. Some perturbations/fluctuations will grow faster than others and dominate the dynamics of phase separation. The wavelength of the fastest growing wave, $\lambda_{m}$, is an important property as it provides a measure of the expected domain size (Figure. 3a). ${ }^{[29]}$

Our framework focuses on the evaporation induced phase separation. It traces the spatial and temporal distribution of the volume fraction of components in thinning films. We study the 
interplay between evaporation kinetics of the solvent from the top surface and diffusion within the film, accompanied by phase separation. The physicochemical properties of the blend components include solubility parameters, ${ }^{[38]}$ viscosity, ${ }^{[37,39]}$ and interaction parameters (FloryHuggins interactions, $\chi_{i j}{ }^{[40]}$ between components. Interaction parameters are key parameters affecting the phase separation and defining the energy landscape. The Flory Huggins energy is very often used to explain the vertical phase separation in polymer blends. ${ }^{[41]}$ The parameters are measured and/or calculated as shown in Table 1 (see Supporting Information for more details). The solubility parameters exhibit a high value of polar and hydrogen components for diF-TES-ADT and a high value of dispersive component for PTAA. The specific viscosity is 5-times higher for the neat PTAA solution as compared with the neat diF-TES-ADT solution, indicating diF-TES-ADT fluid has lower resistance to shear stress during spin-coating ${ }^{\left[{ }^{42]}\right.}$ and consequently a higher possibility of flowing radially and being ejected off the substrate, in agreement with the observations made in Figure 2c. The interaction parameters $\left(\chi_{i j}\right)$ have values of $6.78,4.41$, and 2.99 for $\chi_{\text {polymer-molecule, }} \chi_{\text {molecule-solvent, }}$ and $\chi_{\text {polymer-solvent, }}$ respectively. The solubilities indicate that diF-TES-ADT is a little further apart in solubility space than PTAA, and PTAA:diF-TES-ADT composites are highly immiscible. The high value of interaction parameters indicate strong tendency of the blend to phase separation. Indeed, one observes from the ternary phase diagram (Figure 3a) that the system is unstable within the bulk solution for most configurations, including cases with relatively high content of the solvent. 

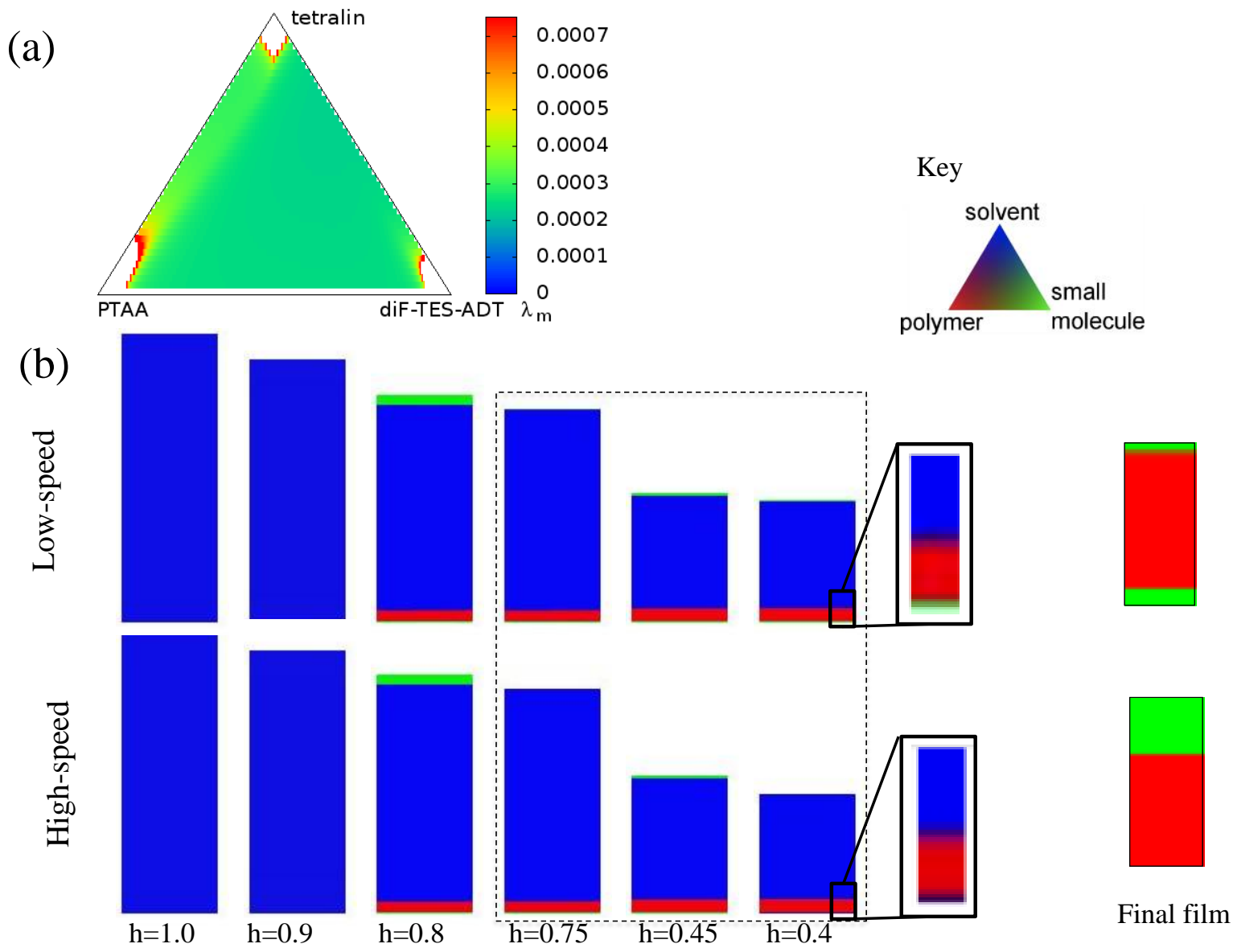

Figure 3. (a) Constructed ternary phase diagram of tetralin, PTAA and diF-TES-ADT blend showing thermodynamics of the system. The colors represent the wavelengths of the fastest growing spinodal instability. ${ }^{[25]}$ Notice that most of the configurations are unstable; (b) Simulations results showing morphology evolution at selected solution film thickness for both slow and high-speed spinning processes. The initial evolution is affected by both centrifugal forces as well as evaporation. In later stages, centrifugal forces are balanced by film viscosity (viscous forces). Morphology evolution in this stage (marked in the dashed square) is dominated by the interplay between evaporation induced and diffusion driven phase separation. For both spinning rates the small molecule diffuses towards the top surface in response to the preferential ejection of the top diF-TES-ADT layer. Small molecule prefers to diffuse to the top in response to preferential loss of diF-TES-ADT, resulting in less diffusion to the buried diF-TES-ADT through the PTAA. This affects the thickness of the buried layer and leads to different final number of layers. The normalized height of the film is indicated by the symbol $h$. 
Table 1. Calculated Hansen solubility parameters, measured specific viscosity and calculated Flory-Huggins interactions for solvent tetralin, PTAA and diF-TES-ADT.

\begin{tabular}{|c|c|c|c|c|c|c|}
\hline \multirow[t]{2}{*}{ Description } & \multicolumn{3}{|c|}{$\begin{array}{l}\text { Hansen solubility parameters } \\
\left(\mathrm{cal} / \mathrm{cm}^{3}\right)^{1 / 2}\end{array}$} & \multirow[t]{2}{*}{$\begin{array}{l}\text { Specific } \\
\text { viscosity }\end{array}$} & \multicolumn{2}{|c|}{ Flory-Huggins interactions $\chi_{i j}$} \\
\hline & $\delta_{\mathrm{p}}$ & $\delta_{\mathrm{p}}$ & $\delta_{\mathrm{h}}$ & & PTAA & diF-TES-ADT \\
\hline Tetralin & 9.58 & 0.98 & 1.42 & - & 2.99 & 4.41 \\
\hline PTAA & 13.96 & 1.45 & 1.45 & 1.26 & - & 6.78 \\
\hline diF-TES-ADT & 9.70 & 5.70 & 4.75 & 0.19 & 6.78 & - \\
\hline
\end{tabular}

During spin-coating, in addition to continuous evaporation of the solvent, the solution flows radially due to the action of centrifugal force, and the excess flow is ejected off the edge of the substrate. The film continues to thin by ejection until viscous forces balance the centrifugal forces. At this point, evaporation becomes the dominant phenomenon that removes solvent from the system. We utilize this framework to model the evolution of the morphology for two simplified cases, namely low and high spin-speeds (see Supplementary information for more information).

For the low spin-speed process, the long-term solvent evaporation (boiling point of tetralin is $\sim 207{ }^{\circ} \mathrm{C}$ ) grants a prolonged process of liquid-liquid phase separation, which in combination with the low shear stress leads to a relatively balanced loss of the two components and the blend ratio remains close to the initial value of $1: 1$. We simulate the distribution of components during the solution thinning process and illustrate in Figure $\mathbf{3 b}$ the evolution of vertical stratification in solution using RGB colors for a better depiction. Red regions indicate polymer-rich domains, green regions denote small molecule-rich domains, and blue regions correspond to the solvent-rich solution. We observe the formation of trilayered stratification for the low-speed case. Initially, the PTAA and diF-TES-ADT components are diluted in the solution $(h=1.00)$. Phase separation happens when the solution begins thinning, e.g., a clear 
segregation of diF-TES-ADT-rich layer forms toward the top surface and bottom surface at $h=0.8$. Interestingly we also observe a weak tendency of diF-TES-ADT diffusion to the bottom to form a buried layer, despite the higher tendency of the PTAA-solvent composite to segregate toward the bottom surface. Continuous thinning of the solution also leads to formation of the PTAA-rich layer. At a height $h=0.08$ (final film), where the sample almost turns to a solid-like film, the resulting morphology is a trilayer stratification consisting of diF-TESADT/PTAA/diF-TES-ADT, in agreement with our experimental observations.

The computational framework suggests that the formation of the buried diF-TES-ADT layer, which relies on diffusion of diF-TES-ADT through the PTAA layer, is sensitive to spinspeed. The simulation results at higher spin-speed indeed exhibit a bilayer with diF-TES-ADT layer at the top (Figure 3b), in agreement with our experimental observations (Figure 1c). The reasons for the diminishing buried diF-TES-ADT layer can be linked to the spin-speed dependent interplay of evaporation with centrifugal driven ejection. Ejection is a result of unbalanced centrifugal $\left(F_{c}\right)^{[43]}$ and viscous forces $\left(F_{v}\right),{ }^{[44]}$ influencing the flow characteristics of the fluid. Force, $F_{c}$ for spinning conditions is given by:

$$
F_{c}=\text { Mass } * \text { Acceleration }=2 \pi r^{2} \rho h \omega^{2}
$$

Similarly, viscous forces $F_{v}$ for spinning is given by:

$$
F_{v}=\text { Viscous shear stress } * \text { Area }=2 \pi r^{2} \eta r \omega / h
$$

The film thickness, $h$, is given by balancing $F_{c}$ and $F_{v}$ :

$$
h=\sqrt{\frac{\eta}{\rho \omega}}=\sqrt{\frac{v}{\omega}}
$$

Here, $\eta$ is the solution viscosity, $\rho$ is the density, $\omega$ is the angular velocity, $r$ is the radius, $h$ is the solution height, and $v$ is the dynamic viscosity. This simple analysis highlights the importance of fluid viscosity in determining the height of the fluid film in the high-speed case. In conjunction with phase separation, the component with lower viscosity is expected to become thinner due to flow and ejection. This clearly points to a higher tendency of the diF-TES-ADT- 
rich layer to be lost due to its lower viscosity in comparison to the PTAA-rich phase. This result is consistent with Norrman's rule ${ }^{[45]}\left(h=k_{2} \eta^{\beta} \omega^{\alpha}\right.$, where $k_{2}$ is an empirically determined constant, while the exponents $\alpha$ and $\beta$ are $\sim-0.5$ and in the range of $0.29-0.39$, respectively).

In an effort to computationally emulate the dynamical spinning experiment, we assumed that the top diF-TES-ADT rich layer (which has a lower viscosity) is ejected upon accelerating to high speed. The simulation indicates that the diF-TES-ADT-rich layer separates once more from the bulk solution. Moreover, small molecule diffuses from the bottom surface towards the top of the wet film, compensating for the loss of diF-TES-ADT, as marked in the dashed square (Figure 3b). This classic signature of coarsening, thus depletes the buried diF-TES-ADT layer, resulting in a bilayer structure with diF-TES-ADT on top of PTAA, in agreement with our experiments. The computational model, explaining the mechanism behind the observed morphology evolution, is comparable with the intuitive explanation offered by Arias et. al., ${ }^{[46]}$ who link the properties of the components with the preferential segregation while limit the work to discussing this hypothesis. The computational framework indicates such preferential segregation is the competing effect of formulation thermodynamics and process kinetics, which links physicochemical properties of components and kinetics of the phase separation, and finally accounts for the preferential loss of one component.

\subsection{Promoting trilayer stratification and carrier transport in the buried OSC layer}

The morphology simulations, in combination with the in situ experimental insight provide us with a better understanding of how liquid-liquid phase separation in unstable formulations, combined with preferential ejection of the small molecule OSC influence the phase separation mechanism in blends. Based on the insight about the importance of OSC volume fraction in the bulk solution for promoting its diffusion toward the buried interface and the experimental observation that the polymer can aggregate earlier in conditions of high 
spinning speeds, we believe that conditions less favorable to preferential ejection of diF-TESADT should promote the formation of a thicker buried OSC layer, which makes the latter more likely to form tight grain and domain boundaries. We have therefore performed very slow spincoating experiments at $300 \mathrm{rpm}$ for duration of $20 \mathrm{~s}$ with the aim of reducing loss of diF-TESADT during spinning and improving the thickness and potentially the crystalline quality of the buried diF-TES-ADT layer in the trilayer thin-film.

(a)

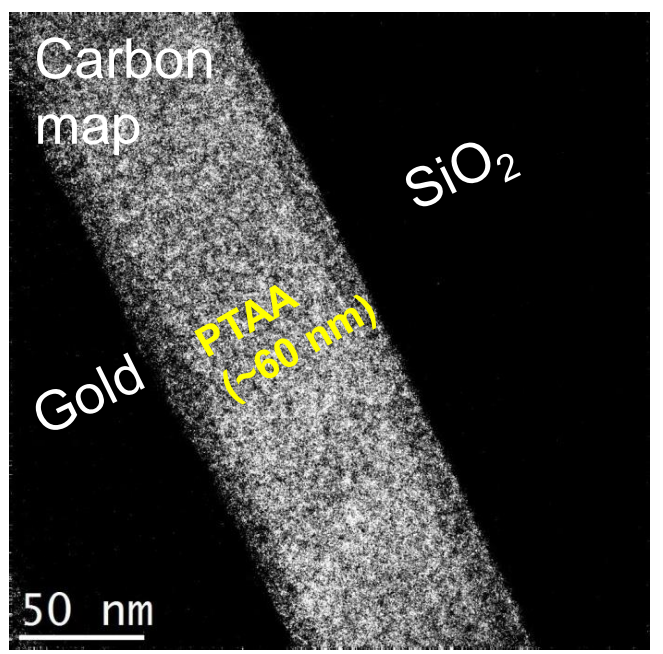

(b)

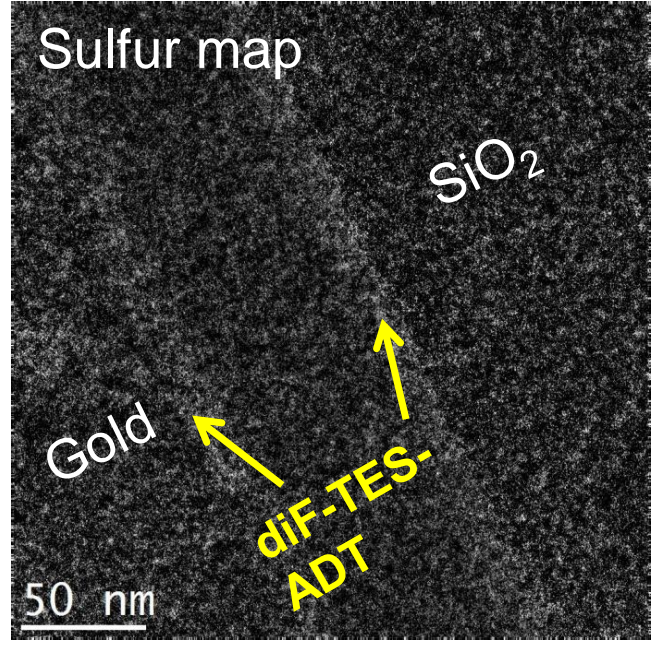

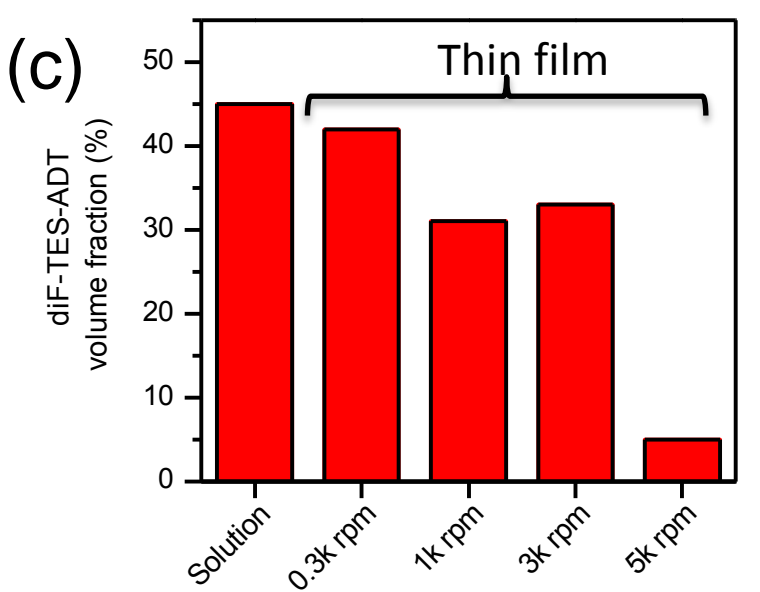

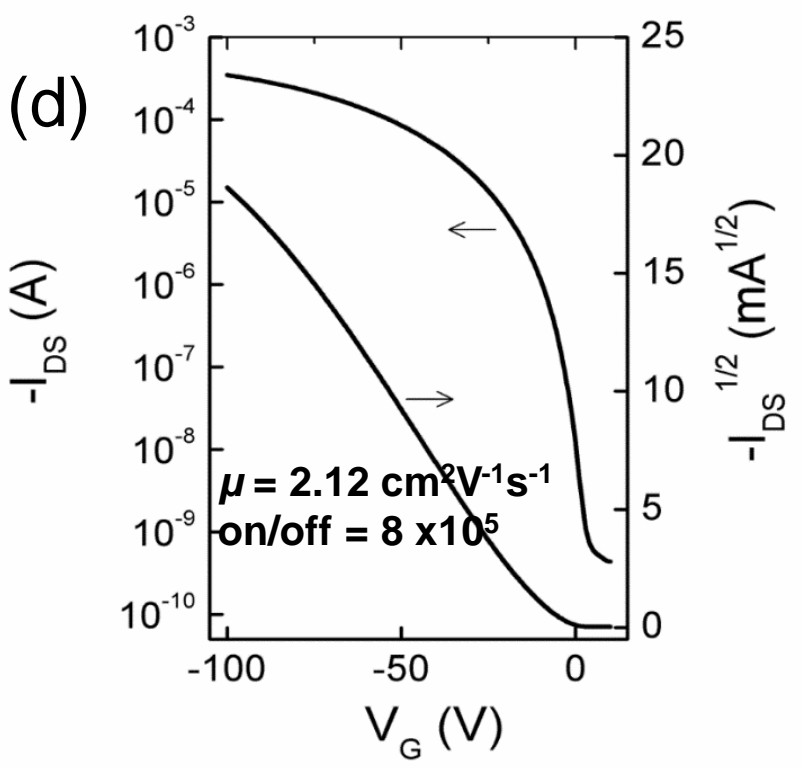

Figure 4. (a) The carbon mapping (284 eV) and (b) sulfur mapping (165 eV) of cross-sectional energy-filtered transmission electron micrographs (EFTEM) for diF-TES-ADT:PTAA film processed at $300 \mathrm{rpm}$ for a duration of $20 \mathrm{~s}$. (c) The diF-TES-ADT volume fraction calculated based on thickness and coverage of diF-TES-ADT, assuming phase purity. The coverage 
calculation was shown in Figure S10. (d) Transfer characteristics ( $I_{D S}$ vs. $V_{G}$ ) of BGTC devices for diF-TES-ADT:PTAA film processed at $300 \mathrm{rpm}$ for a duration of $20 \mathrm{~s}$ on $300 \mathrm{~nm} \mathrm{SiO}_{2}$ and W/L of 10. Tri-layer structure shows diF-TES-ADT ( 22 nm) / PTAA ( 60 nm) / diF-TESADT ( 22 nm). The on/off ratio and mobility reach the high values of $8 \times 10^{5}$ and $2.12 \mathrm{~cm}^{2} \mathrm{~V}^{-}$ ${ }^{1} \mathrm{~S}^{-1}$

In Figure 4 we show the resulting trilayer film structure and the corresponding BGTC device performance, which prove this approach to be successful. The cross-sectional EFTEM reveals a clear trilayer stratification with symmetric $\sim 22 \mathrm{~nm}$ diF-TES-ADT layers at the top and bottom with a $\sim 60 \mathrm{~nm}$ sandwiched PTAA layer. In comparison to the $1000 \mathrm{rpm}$ case, the buried OSC thin film from $300 \mathrm{rpm}$ exhibits very similar thickness for the PTAA layer, but substantially thicker buried and top diF-TES-ADT layers (Figure 4a,b). The volume fraction of diF-TES-ADT estimated from the layer thicknesses and coverage obtained from EFTEM analysis are shown in Figure 4c (see Figure S10 for calculation details). As expected, we find the volume ratio of diF-TES-ADT to PTAA in the $300 \mathrm{rpm}$ dry film to be very close to that of the starting solution, demonstrating that we have indeed nearly avoided preferential ejection of the OSC diF-TES-ADT. The corresponding BGTC device exhibits substantially high carrier mobility up to $2.12 \mathrm{~cm}^{2} \mathrm{~V}^{-1} \mathrm{~s}^{-1}$ with an on/off ratio of $8 \times 10^{5}$ (Figure $4 \mathbf{d}$ ), among the highest reported carrier mobilities for any BGTC device using diF-TES-ADT or any other small molecule OSCs. ${ }^{[26]}$

\section{Conclusion}

In summary, we have demonstrated the importance of processing parameters to mediating vertical stratification and formation of a high quality buried diF-TES-ADT layer for a small molecule (dif-TES-ADT):polymer (PTAA) blend system. This was acquired by performing in- 
situ experimental analysis to monitor solution thinning behavior, fluid state, phase transformation kinetics during spin-coating process, as well as developing a computational framework to predict morphology evolution based on physicochemical properties of blends and phase field-based model. The results indicate PTAA:diF-TES-ADT composites are highly immiscible. Phase separation occurs during solution-casting with diF-TES-ADT-rich layer preferentially segregating on the top and bottom surfaces. The high speed spinning leads to a preferential ejection of the top diF-TES-ADT-rich layer, which prevents mass diffusion of diFTES-ADT onto the bottom surface to form the buried OSC layer. Reducing the fluid dynamic causes for preferential loss of the OSC maintains the solution composition and ultimately promotes trilayer vertical stratification with the polymer sandwiched between two OSC layers. Using this insight, we demonstrate how to alter the processing dynamics to promote the formation of a high quality buried small molecule OSC layer capable of achieving mobility $>2$ $\mathrm{cm}^{2} \mathrm{~V}^{-1} \mathrm{~s}^{-1}$ in the bottom-gate top-contact configuration (BGTC), among the highest reported carrier mobilities reported for any BGTC device using diF-TES-ADT.

\section{Acknowledgments}

Part of this work was supported by the KAUST Office of Competitive Research under the Competitive Research Grants (round 1) program. AA is grateful to SABIC for the Career Development SABIC chair. BG acknowledges partial support from NSF 1435587.

Received: ((will be filled in by the editorial staff))

Revised: ((will be filled in by the editorial staff)) Published online: ((will be filled in by the editorial staff))

\section{Experimental parts}

\subsection{Materials and sample preparation: 2,8-Difluoro-5,11-bis(triethylsilylethynyl)} anthradithiophene (diF-TES-ADT) and poly(bis(4-phenyl)(2,4-dimethylphenyl) amine) 
(PTAA) were prepared by the reported procedures. ${ }^{[1]}$ Single crystal Si (100) wafers with a thermal oxide layer of $300 \mathrm{~nm}$ thickness were used as substrate for the thin film deposition and bottom-gate top-contact OTFTs fabrication. Substrates were cleaned by rinsing with acetone, isopropanol, ethanol, and Milli Q followed by Standard Clean 1 (RCA) ammonium hydroxide $\left(30 \% \mathrm{NH}_{4} \mathrm{OH}\right)$, hydrogen peroxide $\left(30 \% \mathrm{H}_{2} \mathrm{O}_{2}\right)$ and Milli Q (with 1:1:5 ratio) for 15 min at 70 ${ }^{\circ} \mathrm{C}$. Finally all substrates were dried with $\mathrm{N}_{2}$ and heated at $100{ }^{\circ} \mathrm{C}$ for $10 \mathrm{~min}$. The blend semiconductors were deposited by spin-casting at $700 \mathrm{rpm}$ for $10 \mathrm{sec}$ followed by various spinspeeds (from $1000 \mathrm{rpm}$ to $5000 \mathrm{rpm}$ ) for $20 \mathrm{sec}$ from $4 \mathrm{wt} \%$ 1,2,3,4-tetrahydronaphthalene (tetralin) solutions. After spin-casting, the thin-films were thermal annealed immediately at $100{ }^{\circ} \mathrm{C}$ for $5 \mathrm{~min}$. Gold source-drain electrodes were evaporated through a shadow mask with a channel a channel (W/L) 10. All electrical measurements were performed with a Keithely 4200 Semiconductor Characterization System in glove-box.

\subsection{Characterization:}

In-situ absorption measurements are performed using a F20-UVX spectrometer (Filmetrics, Inc.) equipped with a tungsten halogen and a deuterium light sources (Filmetrics, Inc.) over the wavelength range of interest from $350 \mathrm{~nm}$ to $800 \mathrm{~nm}$. Measurements are performed with an integration time of $0.5 \mathrm{~s}$ per absorption spectrum.

In-situ spectroscopic ellipsometry (SE; M-2000XI, J. A. Woollam Co., Inc) measurements and in situ reflection measurements are performed together in a separate spin-coating experiment to monitor the thinning of the solution and thin film formation during the spinning process. For these reflection-mode measurements, a single crystal silicon wafer (100) with a $300 \mathrm{~nm}$-thick thermal oxide is used as substrate. The reflection spectra are analyzed using the Fast Fourier Transform method, which provides a first order estimate of solution thickness over the thickness range from $\sim 10^{5}$ to $\sim 10^{2} \mathrm{~nm}$ without requiring an elaborate model fit or correction for optical nonidealities, such as the spectral bandwidth of the detector or the non-uniformity of the liquid 
film during the early stages of spin-coating. The SE spectra are obtained at an incidence angle of $70^{\circ}$ from substrate normal.

High-speed polarized optical microscope (HSPOM) was performed at KAUST on a Nikon LV100 microscope and recorded by a Photron SA-3 CMOS camera. The camera was mounted on the microscope with a fixed angle of $30^{\circ}$ between polarizer and analyzer. The images were recorded at a speed of 125 frames per second with a shutter speed of $0.004 \mathrm{~s}$.

Grazing Incidence Wide Angle X-Ray Scattering: GIWAXS measurements were carried out at D-line at the Cornell High Energy Synchrotron Source (CHESS) at Cornell University. A 0.5 $\times 0.1 \mathrm{~mm}$ beam size with a wavelength of $1.23 \AA$ and wide band pass $(1.47 \%)$ was generated from double-bounce multilayer monochromator.

Electron microscopy: A transmission electron microscope operating at $300 \mathrm{kV}$ (Titan Cryo Twin, FEI Company, Hillsboro, OR) was used to acquire cross-sectional micrographs using a 4k x 4k charged couple device (CCD) camera model US4000 and an energy filter model GIF Tridiem from Gatan, Inc. (Gatan Inc., Pleasanton, CA). The GIF was utilized in energy-filtered TEM (EF-TEM) mode to image the Carbon and Sulfur distribution in the sample. The Carbon edge located at $284 \mathrm{eV}$ and Sulfur edge at $165 \mathrm{eV}$ were selected to generate the EF-TEM maps using a 3-window method. Samples were prepared on a Helios 400s focused ion beam (FIB; FEI Company), foils were lifted out in situ using an Omniprobe nanomanipulator (AutoProbe300). Electron beam assisted carbon and platinum deposition was performed on the sample surface to protect the thin film surface against the ion beam bombardment during ion beam milling. Ga ion beam ( $30 \mathrm{kV}, 9 \mathrm{nA})$ was first used to cut the sample from the bulk (30 $\mathrm{kV}, 9 \mathrm{nA}$ ), after which it was attached to a $\mathrm{Cu}$ grid using a lift-out method. The sample was subsequently thinned down to ca. $50 \mathrm{~nm}$ thickness $(30 \mathrm{kV}, 93 \mathrm{pA})$ and cleaned $(2 \mathrm{kV}, 28 \mathrm{pA})$ to get rid of areas of the sample damaged during the thinning process. Samples for plan-view TEM were prepared by delaminating and floating the thin films in water and collecting it using 
Zhao et al., Adv. Func. Mater. 2016

a 400 mesh copper grid. TEM images were further treated treated with Gauss blur filtering and Otsu thresholding, to calculate the coverage of diF-TES-ADT surface (Figure S10). 


\section{References:}

[1] J. Smith, W. Zhang, R. Sougrat, K. Zhao, R. Li, D. Cha, A. Amassian, M. Heeney, I. McCulloch, T. D. Anthopoulos, Adv. Mater. 2012, 24, 2441.

[2] H. Chen, Y. Guo, G. Yu, Y. Zhao, J. Zhang, D. Gao, H. Liu, Y. Liu, Adv. Mater. 2012, 24, 4618.

[3] H. Minemawari, T. Yamada, H. Matsui, J. Tsutsumi, S. Haas, R. Chiba, R. Kumai, T. Hasegawa, Nature 2011, 475, 364.

[4] G. Giri, E. Verploegen, S. C. B. Mannsfeld, S. Atahan-Evrenk, D. H. Kim, S. Y. Lee, H. A. Becerril, A. Aspuru-Guzik, M. F. Toney, Z. Bao, Nature 2011, 480, 504.

[5] Y. Yuan, G. Giri, A. L. Ayzner, A. P. Zoombelt, S. C. B. Mannsfeld, J. Chen, D. Nordlund, M. F. Toney, J. Huang, Z. Bao, Nat. Commun. 2014, 5, 3005.

[6] D. J. Gundlach, J. E. Royer, S. K. Park, S. Subramanian, O. D. Jurchescu, B. H. Hamadani, a J. Moad, R. J. Kline, L. C. Teague, O. Kirillov, C. A. Richter, J. G. Kushmerick, L. J. Richter, S. R. Parkin, T. N. Jackson, J. E. Anthony, Nat. Mater. 2008, 7, 216.

[7] R. Hamilton, J. Smith, S. Ogier, M. Heeney, J. E. Anthony, I. McCulloch, J. Veres, D. D. C. Bradley, T. D. Anthopoulos, Adv. Mater, 2009, 21, 1166.

[8] S. K. Park, D. a. Mourey, S. Subramanian, J. E. Anthony, T. N. Jackson, Appl. Phys. Lett. 2008, 93, 043301.

[9] O. D. Jurchescu, S. Subramanian, R. J. Kline, S. D. Hudson, J. E. Anthony, T. N. Jackson, D. J. Gundlach, Chem. Mater. 2008, 20, 6733.

[10] O. D. Jurchescu, D. A. Mourey, S. Subramanian, S. R. Parkin, B. M. Vogel, J. E. Anthony, T. N. Jackson, D. J. Gundlach, Phys. Rev. B 2009, 80, 085201.

[11] R. J. Kline, S. D. Hudson, X. Zhang, D. J. Gundlach, A. J. Moad, O. D. Jurchescu, T. N. Jackson, S. Subramanian, J. E. Anthony, M. F. Toney, L. J. Richter, Chem. Mater. 2011, 23, 1194. 
[12] J. Kang, N. Shin, D. Y. Jang, V. M. Prabhu, D. Y. Yoon, J. Am. Chem. Soc. 2008, 130, 12273.

[13] Y. S. Chung, N. Shin, J. Kang, Y. Jo, V. M. Prabhu, S. K. Satija, R. J. Kline, D. M. DeLongchamp, M. F. Toney, M. A. Loth, B. Purushothaman, J. E. Anthony, D. Y. Yoon, J. Am. Chem. Soc. 2011, 133, 412.

[14] R. Li, H. U. Khan, M. M. Payne, D. M. Smilgies, J. E. Anthony, A. Amassian, Adv. Funct. Mater. 2013, 23, 291.

[15] K. W. Chou, H. U. Khan, M. R. Niazi, B. Yan, R Li, M M. Payne, J E. Anthony, D-M. Smilgies. A Amassian, J. Mater. Chem. C, 2014, 2, 5681.

[16] H. U. Khan, R. Li, Y. Ren, L. Chen, M. M Payne, U. S Bhansali, D.-M Smilgies, J. E Anthony, A. Amassian, ACS Appl. Mater. Interface, 2013, 5, 2325.

[17] M. B. Madec, D. Crouch, G. R. Llorente, T. J. Whittle, M. Geoghegan, S. G. Yeates, J. Mater. Chem. 2008, 18, 3230.

[18] T. Ohe, M. Kuribayashi, R. Yasuda, A. Tsuboi, K. Nomoto, K. Satori, M. Itabashi, J. Kasahara, Appl. Phys. Lett. 2008, 93, 053303.

[19] J. Smith, R. Hamilton, M. Heeney, D. M. de Leeuw, E. Cantatore, J. E. Anthony, I. McCulloch, D. D. C. Bradley, T. D. Anthopoulos, Appl. Phys. Lett. 2008, 93, 253301.

[20] B. A. Brown, J. Veres, R. M. Anemian, R. T. Williams, S. D. Ogier, S. W. Leeming, WO Patent 2005055248, 2005.

[21] S. D. Ogier, J. Veres, M. Zeidan, Wo Patent 2007082584, 2007.

[22] A. Budkowski, Interfacial phenomena in thin polymer films: phase coexistence and segregation (pp. 1-111). ISBN:978-3-540-48836-1. Springer Berlin Heidelberg (1999).

[23] K. Binder, Acta Polymer., 1995, 46, 204.

[24] C. V. Thompson, Annu. Rev. Mater. Res. 2012. 42, 399.

[25] Y. Yao, J. Hou, Z. Xu, G. Li, Y. Yang, Adv. Funct. Mater. 2008, 18, 1783. 
[26] W. H. Lee, D. Kwak, J. E. Anthony, H. S. Lee, H. H. Choi, D. H. Kim, S. G. Lee, K. Cho, Adv. Funct. Mater. 2012, 22, 267.

[27] N. Shin, J. Kang, L. J. Richter, V. M. Prabhu, R. J. Kline, D. A. Fischer, D. M. DeLongchamp, M. F. Toney, S. K. Satija, D. J. Gundlach, B. Purushothaman, J. E. Anthony, D. Y. Yoon, Adv. Funct. Mater. 2013, 23, 366.

[28] O. Wodo, B. Ganapathysubramanian, J. Comput. Phys. 2011, 230, 6037.

[29] O. Wodo, B. Ganapathysubramanian. Appl. Phys. Lett. 2014, 105, 153104.

[30] O. Wodo, B. Ganapathysubramanian, Comput. Mater. Sci. 2012, 55, 113.

[31] A. Amassian, P. Desjardins, L. Martinu, Thin Solid Films, 2004, 447 -448, 40.

[32] M. Abdelsamie, K. Zhao, M. R. Niazi, K. W. Chou, A. Amassian, J. Mater. Chem. C, 2014, 2, 3373.

[33] K. W. Chou, B. Yan, R. Li, E. Q. Li, K. Zhao, D. H. Anjoum, S. Alvarez, R. Gassaway, A. Biocca, S. T. Thoroddsen, A. Hexemer, A. Amassian, Adv. Mater. 2013, 25, 1923.

[34] N. Sahu, B. Parija, S. Panigrahi, Indian J. Phys. 2009, 83, 493.

[35] A. Intaniwet, C. A. Mills, M. Shkunov, H. Thiem, J. L. Keddie, P. J. Sellin, J. Appl. Phys. 2009, 106, 064513.

[36] G. Giri, R. Li, D.-M. Smilgies, E. Qiang Li, Y. Diao, K.a M. Lenn, M. Chiu, D. W. Lin, R. Allen, J. Reinspach, S. C.B. Mannsfeld, S. T. Thoroddsen, P. Clancy, Z. Bao, A. Amassian. Nat. Commun. 2014, 5, 3573.

[37] F. P. V. Koch, J. Rivnay, S. Foster, C. Müller, J. M. Downing, E. B. Domingo, P. Westacott, L. Yu, M. J. Yuan, M. Baklar, Z. P. Fei, C. Luscombe, M. A. McLachlan, M. Heeney, G. Rumbles, C. Silva, A. Salleo, J. Nelson, P. Smith, N. Stingelin, Prog. Polym. Sci. 2013, $38,1978$.

[38] A. F. M. Barton, "CRC handbook of solubility parameters and other cohesion parameters" 2nd ed., CRC Press, Boca Raton, FL 1991.

[39] H. Eyring, T. Ree, N. Hirai, Proc. Natl. Acad. Sci. U. S. 1958, 44, 1213. 
[40] M. K. Chaudhury, G. M. Whitesides, Langmuir 1991, 7, 1013.

[41] S. Nilsson, A. Bernasik, A. Budkowski, E. Moons, Macromolecules, 2007, 40, 8291.

[42] K. R. Symon, “Mechanics”, Third ed, Addison-Wesley Press. 1971, ISBN 0-201-073927.

[43] Y. Pauleau, (Ed). "Chemical physics of thin film deposition processes for micro-and nanotechnologies.” 2012, Vol. 55. Springer Science \& Business Media.

[44] D. B. Hall, P. Underhill, J. M. Torkelson, Polym. Eng. Sci. 1998, 38, 2039.

[45] K. Norrman, A. Ghanbari-Siahkali, N. B. Larsen, Annu. Rep. Prog. Chem., Sect. C. 2005, $101,174$.

[46] A. C. Arias, F. Endicott, R. A. Street, Adv. Mater. 2006, 18. 2900. 